\title{
Generation of Time Delays: Simplified Models of Intracellular Signalling in Cerebellar Purkinje Cells
}

\author{
Volker Steuber \\ Department of Physiology \\ University College London \\ Gower Street \\ London WC1E 6BT \\ $\mathrm{UK}$ \\ David Willshaw \\ Institute for Adaptive and Neural Computation \\ School of Informatics \\ University of Edinburgh \\ 5 Forrest Hill \\ Edinburgh EH1 2QL \\ UK \\ Arjen van Ooyen \\ Center for Neurogenomics and Cognitive Research (CNCR) \\ Department of Experimental Neurophysiology \\ Vrije Universiteit Amsterdam \\ De Boelelaan 1085 \\ $1081 \mathrm{HV}$ Amsterdam \\ The Netherlands
}

Running title: Generation of Time Delays.

Corresponding author: Volker Steuber, Department of Physiology, University College London, Gower Street, London WC1E 6BT, UK, Telephone: +44 20 76793203, Fax: +44 20 79168522, E-mail: v.steuber@ucl.ac.uk. 


\title{
Generation of Time Delays: Simplified Models of Intracellular Signalling in Cerebellar Purkinje Cells
}

\begin{abstract}
In many neuronal systems, information is encoded in temporal spike patterns. The recognition and storage of temporal patterns requires the generation and modulation of time delays between inputs and outputs. In cerebellar Purkinje cells, stimulation of metabotropic glutamate receptors $(\mathrm{mGluRs})$ results in a delayed calcium and voltage response that has been implicated in classical conditioning and temporal pattern recognition. Here, we analyse and simplify a complex model of the intracellular signalling network that has been proposed as a substrate for this delayed response. We systematically simplify the original model, present a minimal model of time delay generation, and show that a delayed response can be produced by the combination of negative feedback and autocatalysis, without any intervening signalling steps that would contribute additive delays. The minimal model is analysed using phase plane methods, and classified as an excitable system. We discuss the implication of excitability for computations performed by intracellular signalling networks in general.
\end{abstract}




\section{Generation of Time Delays: Simplified Models of Intracellular Signalling in Cerebellar Purkinje Cells}

\section{Introduction}

Many neuronal systems use temporal coding for information processing (e.g. Bialek et al., 1991; Gerstner et al., 1996; Thorpe et al., 1996; Laurent, 1996; Rieke et al., 1997; deCharms \& Zador, 2000; Ahissar \& Arieli, 2001; Huxter et al., 2003). In order to use temporal coding, a system has to be able to generate and control time delays in neuronal interactions. Understanding the generation and control of neuronal time delays is a prerequisite for understanding temporal coding in the brain.

An example of a system where delays could play an important role is the cerebellum. In Purkinje cells in the cerebellar cortex, activation of metabotropic glutamate receptors (mGluRs) by parallel fibre input induces a delayed voltage response that has been attributed to a delayed increase in the cytoplasmic calcium concentration (Batchelor \& Garthwaite, 1993; Batchelor et al., 1994; Batchelor \& Garthwaite, 1997; Tempia et al., 1998; Finch \& Augustine, 1998). The delay between mGluR stimulation and calcium response can vary considerably among cells, and this variation in delays has been suggested to underlie the adaptive timing of the classically conditioned eye-blink response (Fiala et al., 1996; Steuber \& Willshaw, 1997, 2004) and the recognition of temporal parallel fibre patterns (Steuber \& Willshaw, 1999, 2004). In other systems than the cerebellum, delays have also been implicated in processes such as sound localisation and temporal pattern recognition in general (Hopfield, 1995; Gerstner et al., 1996; Napp-Zinn et al., 1996; Eurich et al., 1997, 1998; Hüning et al., 1998; Natschläger \& Ruf, 1998).

The signalling cascade involved in generating the delay between the mGluR activation and the $\mathrm{Ca}^{2+}$ response in Purkinje cells has not yet been fully characterised, but seems to require $\mathrm{Ca}^{2+}$ release from intracellular stores (Finch \& Augustine, 1998). A plausible hypothesis is that the delay is based on mGluR evoked activation of phospholipase C, generation of inositol $(1,4,5)$-trisphosphate $\left(\mathrm{IP}_{3}\right)$ and $\mathrm{IP}_{3}$ induced $C a^{2+}$ release. This hypothesis has been formalised in a complex mathematical model of the mGluR signalling cascade (Fiala et al., 1996). Using their model, Fiala and collaborators showed that 
this signalling cascade can indeed generate a wide spectrum of delays between receptor activation and response.

However, Fiala et al. did not pinpoint which interactions in the complex mGluR signalling network are responsible for generating the delay. A possible explanation for the delayed response could be that the delay is simply caused by the sum of the time delays that are introduced by the successive signalling steps between mGluR stimulation and $\mathrm{Ca}^{2+}$ response. A similar kind of additive mechanism has been used by Goldbeter to generate a time delay between mRNA production and negative feedback in a model of circadian oscillations of the Drosophila PER protein (Goldbeter, 1995).

Alternatively, the delayed response might not be based on the sum of individual delays, but emerge from the set of specific interactions between a few components of the mGluR signalling cascade. The full model is too complex to be able to exclude either of these two alternatives. In order to identify the minimal requirements for the generation of the delayed response, we simplify the Fiala model in several steps, from the original system of 12 ordinary differential equations (ODEs) first down to nine equations, then to five and finally to a minimal model of two ODEs. We show that the simplified model reproduces the behaviour of the full model, including the possibility of generating delays of different lengths, and that the model belongs to a general class of excitable systems. Using our minimal model, we identify $\mathrm{Ca}^{2+}$ dependent autocatalysis and negative feedback between $\mathrm{Ca}^{2+}$ and activated receptors as the essential elements for the generation of the delayed response.

\section{The Fiala Model}

Before presenting our simplifications we first describe the original Fiala model (Fiala et al., 1996, see figure 1 and appendix). Fiala and collaborators suggest that the time delay generated by the intracellular signalling cascade in cerebellar Purkinje cells is important for classical conditioning of eye-blink responses (for review, see Thompson \& Krupa, 1994; Yeo \& Hesslow, 1998). In eye-blink conditioning, repeated conjunctive presentations of a conditioned stimulus (CS, for example a tone) followed by an unconditioned stimulus (US, for example a periorbital shock) result in an association between CS and US. After 
training with paired CS and US, presentations of the CS alone trigger adaptively timed eye-blink conditioned responses (CRs) at the time of the US onset during training.

It has been suggested that the association between CS and US that is formed during eye-blink conditioning is based on a decrease in Purkinje cell responsiveness, which leads to disinhibition of deep cerebellar nucleus neurons and increased output from the cerebellum (see for example Thompson \& Krupa, 1994; Yeo \& Hesslow, 1998). Information about CS and US reaches cerebellar Purkinje cells through parallel fibres (PFs) and climbing fibres (CFs), respectively. In the Fiala model, CS evoked PF fibre input to the Purkinje cell results in stimulation of $\mathrm{mGluRs}$ by glutamate. This activates an intracellular signalling cascade in the Purkinje cell, leading to release of $\mathrm{Ca}^{2+}$ from intracellular stores and an increase of the cytoplasmic $\mathrm{Ca}^{2+}$ concentration. After training, the CS mediated increase in $\mathrm{Ca}^{2+}$ concentration results in an outward current through activated $\mathrm{Ca}^{2+}$ dependent $K^{+}$(KCa) channels, a decrease of the Purkinje cell firing rate and execution of an eyeblink CR.

The time delay between mGluR activation and $\mathrm{Ca}^{2+}$ response varies depending on the density of available mGluRs at the PF synapse. The timing of the $\mathrm{Ca}^{2+}$ response is of central importance in the Fiala model: eye-blink learning is restricted to Purkinje cells where the $\mathrm{Ca}^{2+}$ response coincides with the production of cGMP in the cytoplasm that is triggered by US evoked CF input (Ito \& Karachot, 1992, see appendix). In these cells, the paired presentations of CS and US result in persistent phosphorylation and activation of KCa channels. Fiala and collaborators assume that there is an array of Purkinje cells with $\mathrm{Ca}^{2+}$ response latencies betwen $100 \mathrm{~ms}$ and several seconds. They show that such a Purkinje cell array can learn eye-blink CRs with latencies in the same range.

The network of interactions between the intracellular signalling components in the Fiala model is shown in figure 1. In addition to the feedforward connections linking the activation of mGluRs by glutamate with the change in $\mathrm{Ca}^{2+}$ concentration and membrane voltage there are also several feedback connections. For example, phosphorylation by protein kinase C (PKC) inactivates mGluRs and G-proteins (Nishizuka, 1988; Kawabata et al., 1996; Yarfitz \& Hurley, 1994). $\mathrm{IP}_{3}$ receptors contain stimulating as well as inhibitory binding sites for $\mathrm{Ca}^{2+}$, leading to a biphasic dependence of $\mathrm{Ca}^{2+}$ flux on the cytoplasmic $\mathrm{Ca}^{2+}$ concentration (Bezprozvanny et al., 1991). Furthermore, a $\mathrm{Ca}^{2+}$ ac- 
tivated form of phospholipase C (PLC) provides a positive feedback loop between $\mathrm{Ca}^{2+}$ and $\mathrm{IP}_{3}$ production (Mignery et al., 1992). In the following sections, we simplify the Fiala model in several steps and show that the combination of feedforward and feedback connections can generate the delayed response.

\section{Initial Simplification of the Model}

Our first step towards simplifying the Fiala model is to categorise the components into different groups. First, it is possible to classify the components according to their position in the mGluR signalling network. The earliest of the components that show a delay is $\mathrm{Ca}^{2+}$. As a consequence, only components upstream from $\mathrm{Ca}^{2+}$ can be involved in the generation of the delayed response. Components without any direct or indirect influence on $\mathrm{Ca}^{2+}$ are unable to contribute to the time delay. Thus, cGMP, calcineurin, the maximum $\mathrm{K}_{\mathrm{Ca}}$ channel conductance and the membrane voltage can all be omitted from the model. Only nine components exist whose dynamics can be responsible for the production of the time delay: active mGluRs (B), PKC-inactivated mGluRs (A), G-protein $\mathrm{G}_{P L C \alpha}$ (G), IP3 (I), DAG (D), $C a^{2+}(\mathrm{C}), C a^{2+}$-activated $\mathrm{IP}_{3}$ receptors $\left(\mathrm{R}_{a}\right), C a^{2+}$-inactivated $\mathrm{IP}_{3}$ receptors $\left(\mathrm{R}_{i}\right)$ and $\mathrm{PKC}(\mathrm{P})$.

A second criterion that can be used to classify the components is their qualitative temporal behaviour in response to receptor activation. As can be seen in figure 2, the components can be divided into five different categories:

1. Components that rise quickly (within less than $20 \mathrm{~ms}$ ) up to a plateau, stay constant for a while and decrease rapidly back to the baseline: active mGluRs and glutamate.

2. Components that increase slowly over several hundred milliseconds and, after reaching a peak, decrease quickly: G-protein $\mathrm{G}_{P L C \alpha}$ and $C a^{2+}$-activated $\mathrm{IP}_{3}$ receptors.

3. Components that, after a time delay of several hundred milliseconds, rise quickly, reach a peak with a 10-90\% rise time between 20 and $40 \mathrm{~ms}$ and decay quickly: $\mathrm{Ca}^{2+}, \mathrm{IP}_{3}, \mathrm{DAG}, \mathrm{PKC}$, the membrane voltage ${ }^{1}$, and calcineurin.

\footnotetext{
${ }^{1}$ After training, the voltage response is a negative rather than a positive peak.
} 
4. Components that, after a time delay, rise quickly within 20-40 ms, reach a plateau and decay very slowly (by approximately $10 \%$ over $250 \mathrm{~ms}$ ): PKC-inactivated mGluRs and $\mathrm{Ca}^{2+}$-inactivated $\mathrm{IP}_{3}$ receptors.

5. Components that, during each learning cycle, change by a small amount and keep their new values: the maximum $\mathrm{K}_{C a}$ channel conductance.

Based on the categorisation of the components, the following simplifications can be made:

- $\mathrm{Ca}^{2+}$ and PKC are both category three components with a very similar time course, and $\mathrm{Ca}^{2+}$ precedes PKC directly in the intracellular signalling cascade. As a consequence, PKC can be omitted from the model and, in the simplified equations, the effects of PKC can be represented as $\mathrm{Ca}^{2+}$ effects.

- Similarly, $\mathrm{IP}_{3}$ and $\mathrm{DAG}$ are both category three components and, because of the choice of parameters in the Fiala model, they follow an identical time course. Thus, only $\mathrm{IP}_{3}$ needs to be represented in the simpler version of the model.

- Both glutamate and glutamate-activated mGluRs are category one components. As shown in figure 2 , the mGluR plateau results in a slow increase of the category two component $\mathrm{G}_{P L C \alpha}$. As the temporal evolution of active mGluRs in the cell is not known, a possible simplification of the model is to leave out $\mathrm{G}_{P L C \alpha}$, reduce the rate constant for mGluR activation so that the active mGluRs turn from a category one component into a slowly increasing category two component, and replace all $\mathrm{G}_{P L C \alpha}$ effects by mGluR effects.

- Reducing the rate constant of mGluR activation enables us to make another simplification. Fiala and collaborators assume the existence of three separate receptor pools: activated mGluRs (B), mGluRs that are inactivated by phosphorylation (A), and mGluRs that are dephosphorylated and ready to be activated by glutamate $\left(\mathrm{B}_{\max }-\mathrm{B}-\mathrm{A}\right)$. In their model, the activation of mGluRs is slowed down when the accumulation of phosphorylated receptors leads to a decrease in the concentration of receptors that can be activated. In the simplified model, the rate constant of mGluR activation is more than two orders of magnitude smaller, which ensures 
that the receptor activation is slow enough without having to represent the phosphorylated receptors explicitly as a separate pool. The inactivated mGluRs can be represented collectively by $\left(\mathrm{B}_{\max }-\mathrm{B}\right)$, and the time course of activated receptors $\mathrm{B}$ resembles the time course of activated G-protein $\mathrm{G}_{P L C \alpha}$ in the full model (compare figures $2(\mathrm{~b})$ and $3(\mathrm{a}))$.

The 5 components that are left and whose temporal evolution is described explicitly by ODEs in the simplified model are active mGluRs $(\mathrm{B}), \mathrm{IP}_{3}(\mathrm{I})$, activated $\mathrm{IP}_{3}$ receptors $\left(\mathrm{R}_{a}\right)$, inactivated $\mathrm{IP}_{3}$ receptors $\left(\mathrm{R}_{i}\right)$ and $\mathrm{Ca}^{2+}(\mathrm{C})$. The change of the concentration of active mGluRs in the 5 ODE model is given by:

$$
\frac{d B}{d t}=k_{1}\left(B_{\max }-B\right)[G l u]-k_{-1} B-k_{2} B C
$$

where $[G l u]$ and $B_{\max }$ are the concentrations of glutamate and total available mGluRs, respectively. The third term in equation 1 represents the $\mathrm{Ca}^{2+}(\mathrm{C})$ dependent inactivation of the receptors by $\mathrm{PKC}$.

The activation of the mGluRs leads to an increase in the concentration of $\mathrm{IP}_{3}$, which is modelled by:

$$
\frac{d I}{d t}=\left(I_{\max }-I\right)\left(k_{7} B+k_{8} \frac{C^{2}}{C^{2}+K_{C}}\right)-k_{9} I
$$

As in the Fiala model, the term $k_{8} C^{2} /\left(C^{2}+K_{C}\right)$ describes the $\mathrm{IP}_{3}$ production by a $\mathrm{Ca}^{2+}$ dependent form of phospholipase C.

$\mathrm{IP}_{3}$ binds to $\mathrm{IP}_{3}$ receptor $\mathrm{Ca}^{2+}$ channels on the membrane of the endoplasmic reticulum. Required for the opening of the $\mathrm{IP}_{3}$ receptors is the binding of $\mathrm{IP}_{3}$ and the binding of $\mathrm{Ca}^{2+}$ to an activation site. In the model, the change in the concentration of $\mathrm{Ca}^{2+}$ activated $\mathrm{IP}_{3}$ receptors $R_{a}$ is given by:

$$
\frac{d R_{a}}{d t}=k_{12}\left(R_{\max }-R_{a}-R_{i}\right) C-k_{13} R_{a}-k_{14} R_{a} C^{n}+k_{15} R_{i}
$$

Activated $\mathrm{IP}_{3}$ receptors can be inactivated by dissociation of $\mathrm{Ca}^{2+}$ or by binding of $\mathrm{Ca}^{2+}$ to an inhibitory site. The $\mathrm{Ca}^{2+}$ inactivation of the receptors exhibits a cooperativity of $n=1.65$ (Meissner et al., 1986), and the change of inactivated receptors $R_{i}$ is described by: 


$$
\frac{d R_{i}}{d t}=k_{14} R_{a} C^{n}-k_{15} R_{i}
$$

The binding of $\mathrm{IP}_{3}$ is assumed to be in equilibrium with respect to the binding and dissociation of $\mathrm{Ca}^{2+}$, and the concentration of open $\mathrm{IP}_{3}$ receptor channels $R_{o}$ is given by:

$$
R_{o}=\frac{I}{I+K_{I}} R_{a}
$$

The release of $\mathrm{Ca}^{2+}$ through the open $\mathrm{IP}_{3}$ receptor channels $R_{o}$ leads to an increase in the cytoplasmic $\mathrm{Ca}^{2+}$ concentration $C$ :

$$
\frac{d C}{d t}=k_{16} R_{o}\left(C_{E R}-C\right)-k_{17} \frac{C^{2}}{C^{2}+K_{\text {ATPase }}}
$$

where $C_{E R}$ is the $C a^{2+}$ concentration in the endoplasmic reticulum. The second term in equation 6 describes the removal of $C a^{2+}$ by the endoplasmic $C a^{2+}$ ATPase. In contrast to the Fiala model, the 5 ODE model is not concerned with the electrogenic effects of the $\mathrm{Ca}^{2+}$ currents, and the $\mathrm{Na}^{+} / \mathrm{Ca}^{2+}$ exchanger flux is therefore not modelled.

The 5 ODE model was implemented in $\mathrm{C}++$ and the equations integrated numerically, using a 5th order Runge-Kutta algorithm with adaptive step size control and the parameter values listed in table 1 . Simulation results for the application of a $500 \mathrm{~ms}$ glutamate pulse to a model with a constant concentration of available mGluRs $B_{\max }=20 \mu M$ are shown in figure 3. The glutamate pulse results in a slow increase in the concentration of active mGluRs, which in turn leads to a very slow increase in the concentrations of $\mathrm{IP}_{3}$ and $\mathrm{Ca}^{2+}$. After a time delay of approximately $250 \mathrm{~ms}$, the $\mathrm{IP}_{3}$ and $\mathrm{Ca}^{2+}$ concentrations begin to rise very quickly, and the model "fires" an $\mathrm{IP}_{3}$ peak and a $\mathrm{Ca}^{2+}$ peak, both of which reach their maxima at $t \approx 300 \mathrm{~ms}$. A consequence of the $C a^{2+}$ rise is a fast decrease in the concentrations of active $\mathrm{mGluRs}$ and $\mathrm{IP}_{3}$ receptors.

To understand the mechanism of the delayed response, it is crucial to understand the transition between the slow rise of $\mathrm{IP}_{3}$ and $\mathrm{Ca}^{2+}$ during the first several hundred milliseconds of glutamate application, and the fast rise during the upstrokes of the peaks. A possible reason for this transition could be that the concentrations of $\mathrm{IP}_{3}$ or $\mathrm{Ca}^{2+}$ reach a threshold where one or several regenerative processes lead to a large increase in the $\mathrm{IP}_{3}$ production and the $\mathrm{Ca}^{2+}$ release rates. In general, such a regenerative process could be a direct autocatalytic effect of a single component on its own formation, or an indirect 
autocatalytic or cross catalytic interaction between two components, meaning that the first component has a positive influence on the formation of the second component and vice versa. Two examples for indirect autocatalysis can be found in the model (compare figures 1 and 4):

1. $\mathrm{Ca}^{2+}$ has a positive effect on the formation of $\mathrm{IP}_{3}$, and $\mathrm{IP}_{3}$ stimulates release of $\mathrm{Ca}^{2+}$ into the cytoplasm.

2. $\mathrm{Ca}^{2+}$ binds to and activates $\mathrm{IP}_{3}$ receptors, which leads to release of $\mathrm{Ca}^{2+}$ into the cytoplasm.

Although the existence of autocatalysis might be able to explain the upstrokes of the peaks, another mechanism is needed to generate the downstrokes, which reset the $\mathrm{IP}_{3}$ and $\mathrm{Ca}^{2+}$ concentrations back to their baseline values. A possible reset mechanism could be the $\mathrm{Ca}^{2+}$ dependent negative feedback through PKC phosphorylation of the active mGluRs (see equation 1 and figures 1 and 4 ). To investigate if the combination of autocatalysis and negative feedback is sufficient for the generation of the delayed response, the 5 ODE model was further simplified down to a minimal model with 2 ODEs.

\section{The Minimal Model}

In the minimal model, the dynamics of activated mGluRs $B$ and cytoplasmic calcium $C$ are described by two equations:

$$
\begin{gathered}
\frac{d B}{d t}=k_{a}\left(B_{\max }-B\right)[G l u]-k_{b} B-k_{c} B f_{a}(C) \\
\frac{d C}{d t}=k_{d} B f_{b}(C)-k_{e} f_{c}(C)
\end{gathered}
$$

where $B_{\max }$ is again the total concentration of available mGluRs. The three different terms in equation 7 represent the activation of inactive mGluRs $\left(B_{\max }-B\right)$ by glutamate, the inactivation of active mGluRs by dissociation of glutamate and their $\mathrm{Ca}^{2+}$ dependent inactivation by PKC phosphorylation. These terms correspond directly to those in the original Fiala model, with the exception that, like in the 5 ODE model, active PKC 
is not modelled explicitly and the model has been simplified by making the receptor phosphorylation directly dependent on $\mathrm{Ca}^{2+}$.

The two terms in equation 8 represent the release of $\mathrm{Ca}^{2+}$ from intracellular stores and the removal of $C a^{2+}$ from the cytoplasm. The first term $\left(k_{d} B f_{b}(C)\right)$ summarises all steps connecting the activated mGluRs with the influx of $\mathrm{Ca}^{2+}$ into the cytoplasm, including G-protein activation, production of $\mathrm{IP}_{3}$ and DAG by PLC, activation of $\mathrm{IP}_{3}$ receptors by $\mathrm{IP}_{3}$ and $\mathrm{Ca}^{2+}$ flux through the $\mathrm{IP}_{3}$ receptors (in the Fiala model and the 5 ODE model, these processes are modelled by equations 20 - 32 and equations 2 - 6, respectively, see appendix and previous section). The production of $\mathrm{IP}_{3}$ by PLC and the activation of the $\mathrm{IP}_{3}$ receptors are $C a^{2+}$ dependent; this is reflected by the term $f_{b}(C)$. The second term in equation 8 represents the active transport of $\mathrm{Ca}^{2+}$ out of the cytoplasm and corresponds directly to equation 29 in the Fiala model and equation 6 in the 5 ODE model.

In the previous section, we suggested that the time delay between receptor stimulation and $\mathrm{Ca}^{2+}$ response might be based on the combined action of negative feedback and autocatalysis. The following scenario could be responsible for the delayed $\mathrm{Ca}^{2+}$ peak:

1. Initially, the glutamate pulse leads to a slow increase in the concentration of active receptors $B$ and a very slow increase in the $C a^{2+}$ concentration $C$. As long as $C$ is smaller than a threshold concentration $\Theta_{a}$, neither autocatalysis nor negative feedback have a significant effect, and the rate of $\mathrm{Ca}^{2+}$ uptake back into the stores is very low.

2. When $C$ reaches $\Theta_{a}$, the autocatalysis is switched on, leading to a large increase in the $\mathrm{Ca}^{2+}$ release rate and the onset of the $\mathrm{Ca}^{2+}$ peak.

3. On the way to the top of the peak, $C$ passes a second threshold concentration $\Theta_{b}$ which results in a large increase in the $\mathrm{Ca}^{2+}$ uptake rate.

4. After $C$ reaches a third threshold $\Theta_{c}$, the negative feedback is activated and the concentration of active receptors $B$ is beginning to fall.

5. Consequence of the decrease in $B$ is a decrease in the $C a^{2+}$ release rate until $\mathrm{Ca}^{2+}$ release and uptake compensate each other and the maximum of the peak is reached. 
6. The continued decrease in the rate of release of $\mathrm{Ca}^{2+}$ leads to the downstroke of the peak and the return of the $\mathrm{Ca}^{2+}$ concentration to the baseline.

In the full model, the calcium dependent negative feedback, autocatalysis and removal of calcium from the cytoplasm, which are summarised by the three functions $f_{i}(C)$, contain sigmoidal dependencies on the calcium concentration. In the simplified model, these $\mathrm{Ca}^{2+}$ dependencies are represented by three Hill functions:

$$
f_{i}(C)=\frac{C^{n}}{C^{n}+K_{i}^{n}}
$$

When the cooperativity $n$ is larger than one, a Hill function describes a sigmoid with a steepness determined by $n$ and a half-maximal value for $C=K_{i}$. Thus, in the model with three Hill functions, the three constants $K_{i}$ correspond to the threshold concentrations $\Theta_{a}, \Theta_{b}$ and $\Theta_{c}$ for the three $C a^{2+}$ dependent processes.

Compared to the original 12 ODE Fiala model and our 9 and 5 ODE models, the 2 ODE model can be much more easily analysed, and it is possible to display the temporal evolution of the complete system in the phase plane. A first step towards analysing the behaviour of the system is to determine the nullclines where the temporal derivative of one of the variables is zero, and the fixed points where the temporal derivatives of both variables are zero. In the 2 ODE model, the set of coordinates $\{B, C\}$ that satisfy $\dot{B}=0$ defines a single B-nullcline:

$$
B=\frac{k_{a}[G l u] B_{\max }\left(C^{n}+K_{a}^{n}\right)}{k_{a}[G l u]\left(C^{n}+K_{a}^{n}\right)+k_{b}\left(C^{n}+K_{a}^{n}\right)+k_{c} C^{n}}
$$

The model has two C-nullclines describing the coordinates $\{B, C\}$ which are given by $\dot{C}=0$. The first of the C-nullclines is the B-axis, $C=0$, and the second C-nullcline is given by:

$$
B=\frac{k_{e}\left(C^{n}+K_{b}^{n}\right)}{k_{d}\left(C^{n}+K_{c}^{n}\right)}
$$

The intersections of the B-nullcline with one of the two C-nullclines are the fixed points of the system. For all possible combinations of parameter values, the B-nullcline intersects the B-axis exactly once. At this intersection, the system has a fixed point: 


$$
\left\{\bar{B}_{1}, \bar{C}_{1}\right\}=\left\{\frac{k_{a}[G l u] B_{\max }}{k_{a}[G l u]+k_{b}}, 0\right\}
$$

Depending on the choice of parameters in the model, the B-nullcline can also intersect the second C-nullcline (equation 11) at a single location. Thus, the model can have a second fixed point $\left\{\bar{B}_{2}, \bar{C}_{2}\right\}$ whose coordinates are:

$$
\begin{gathered}
\bar{B}_{2}=\frac{k_{e}\left(2 K_{b}^{n} \bar{S}-\bar{R}+\sqrt{\bar{R}^{2}-4 \bar{S} \bar{T}}\right)}{k_{d}\left(2 K_{c}^{n} \bar{S}-\bar{R}+\sqrt{\bar{R}^{2}-4 \bar{S} \bar{T}}\right)} \\
\bar{C}_{2}=\left(\frac{-\bar{R}+\sqrt{\bar{R}^{2}-4 \bar{S} \bar{T}}}{2 \bar{S}}\right)^{1 / n}
\end{gathered}
$$

where $\bar{S}, \bar{T}$ and $\bar{R}$ are given by:

$$
\begin{gathered}
\bar{R}=k_{a} k_{e} K_{b}^{n}[G l u]+k_{a} k_{e} K_{a}^{n}[G l u]+k_{b} k_{e} K_{b}^{n}+k_{b} k_{e} K_{a}^{n} \\
+k_{c} k_{e} K_{b}^{n}-k_{a} k_{d} K_{c}^{n}-k_{a} k_{d} K_{a}^{n}[G l u] B_{\max } \\
\bar{S}=k_{a} k_{e}[G l u]+k_{b} k_{e}+k_{c} k_{e}-k_{a} k_{d}[G l u] B_{\max } \\
\bar{T}=k_{a} k_{e} K_{a}^{n} K_{b}^{n}[G l u]+k_{b} k_{e} K_{a}^{n} K_{b}^{n}-k_{a} k_{d} K_{a}^{n} K_{c}^{n}[G l u] B_{\max }
\end{gathered}
$$

Using the GRIND integration package (De Boer, 1983), the behaviour of the model was investigated for different combinations of parameter values. We found that only parameter sets that result in an intersection of the B-nullcline (equation 10) with the second Cnullcline (equation 11), and therefore give rise to the second fixed point $\left\{\bar{B}_{2}, \bar{C}_{2}\right\}$, are able to produce a time delay between the onset of the mGluR activation and the intracellular $\mathrm{Ca}^{2+}$ response.

The existence of this fixed point is necessary for the model to generate a delayed reponse, but it is not sufficient. When searching the parameter space of the model, we could only generate delayed responses for Hill coefficients $n \geq 2$, although parameter sets with $n=1$ can also lead to a stable fixed point at $\left\{\bar{B}_{2}, \bar{C}_{2}\right\}$. These empirical results indicate that the sigmoidal shape of the $\mathrm{Ca}^{2+}$ dependencies favours the generation of time delays in the model. 
Figures 5 and 6 summarise the behaviour of the minimal model for a cooperativity $n=4$ and the parameter values that are given in table 2. As shown in panel 5 (a), the B-nullcline and the second C-nullcline divide the biologically meaningful area of the phase plane where $B$ and $C$ are larger than or equal to zero into four regions with different orientations of the direction vector $\{\dot{B}, \dot{C}\}$. The fixed point $\left\{\bar{B}_{2}, \bar{C}_{2}\right\}$ at the intersection of the B-nullcline and the second C-nullcline is stable, and the fixed point $\left\{\bar{B}_{1}, \bar{C}_{1}\right\}$ at the intersection of the B-nullcline with the B-axis is a saddle point. Thus, if the initial $\mathrm{Ca}^{2+}$ concentration $C(0)$ is equal to zero, the system will travel along the B-axis and end up at $\left\{\bar{B}_{1}, \bar{C}_{1}\right\}$, and if $C(0)$ is different from zero, the system will move through the phase plane and come to rest at $\left\{\bar{B}_{2}, \bar{C}_{2}\right\}$.

The behaviour of the model in response to parallel fibre input is shown in panel 5 (b). Without any input to the cell, the glutamate concentration in the synaptic cleft is at a very low level $G_{1}$, and the system rests in the stable state $\mathbf{S}^{0}=\left\{\bar{B}_{2}, \bar{C}_{2}\right\}$ at the intersection of the B-nullcline and the second C-nullcline (indicated by the empty circle in the figure). When the cell receives an input, the glutamate concentration is temporarily stepped up to a higher value $G_{2} \gg G_{1}$. As a result of the glutamate step, the B-nullcline (equation 10) is shifted in the phase plane, and the stable fixed point moves to a new location $\mathbf{S}^{*}=\left\{\bar{B}_{2}^{*}, \bar{C}_{2}^{*}\right\}$ (filled circle in panel $5(\mathrm{~b})$ ). Thus, the current state of the system $\mathbf{S}^{0}$ is no longer a fixed point, and the state vector $\mathbf{S}=\{\bar{B}, \bar{C}\}$ travels through the phase plane until it settles into the new stable state at $\mathbf{S}^{*}$. The temporal evolution of the system in response to the $G_{1} \rightarrow G_{2}$ step can be divided into the following five phases:

1. A very slow increase in $C$ and an increase in $B$ while the state vector $\mathbf{S}$ crosses the $(++)$ region. The increase in $B$ is initially fast $\left(1.48 \mu M / s\right.$ at $\mathbf{S}^{0}$, see vector field in panel $5(\mathrm{~b}))$ and slows down when the state vector approaches the $B$ nullcline.

2. A very fast increase in $C$ (reaching a maximum rate of $20.09 \mu \mathrm{M} / \mathrm{s}$ ) and a fast decrease in $B$ while crossing the $(-+)$ region.

3. A fast decrease in $C$ and a slow and very small decrease in $B$ on the way through the $(--)$ region.

4. A very small decrease in $C$ and a very small increase in $B$ while traversing the $(+-)$ region. 
5. Finally, a very small increase in $C$ and $B$ while $\mathbf{S}$ crosses the $(++)$ region for a second time before the system settles into the new stable state $\mathbf{S}^{*}$.

Although the high-glutamate fixed point $\mathbf{S}^{*}$ is close to the low-glutamate fixed point $\mathbf{S}^{0}$, the state vector makes a large excursion through the phase plane, a phenomenon which is called excitability (FitzHugh, 1960; Edelstein-Keshet, 1987). The long trajectory in the phase diagram corresponds to a large transient increase in the concentrations of active mGluRs and $\mathrm{Ca}^{2+}$. Two examples of delayed $\mathrm{Ca}^{2+}$ responses for different concentrations of available receptors are given in panels 6 (a) and (b). Panel 6 (c) shows the dependence of the time delay on the concentration of available mGluRs $B_{\max }$. Values of $B_{\max }$ between 30 and $180 \mu M$ result in delayed responses with latencies between 160 and $600 \mathrm{~ms}$. Like in the Fiala model, larger concentrations of available mGluRs give rise to shorter latencies.

When the input to the cell is switched off, the glutamate concentration is reset to the rest value $G_{1}$, and the B-nullcline moves back to its original location. As a consequence, the stable fixed point shifts back to $\mathbf{S}^{0}$ and the current state $\mathbf{S}=\mathbf{S}^{*}$ is destabilised. Thus, the state vector $\mathbf{S}$ travels back to $\mathbf{S}^{0}$, this time without a large detour from the direct route through the phase plane.

\section{Discussion}

In cerebellar Purkinje cells, stimulation of metabotropic glutamate receptors (mGluRs) can result in a cytoplasmic calcium rise and a voltage response after a delay of several hundred milliseconds (Batchelor \& Garthwaite, 1993; Batchelor et al., 1994; Batchelor \& Garthwaite, 1997; Tempia et al., 1998; Finch \& Augustine, 1998). In order to identify the features of the mGluR activated signalling cascade that are critical for generating this delayed response, as well as delayed responses in general, we have analysed and simplified the Fiala model of mGluR dependent intracellular signalling in Purkinje cells (Fiala et al., 1996). Based on the position of the components in the signalling network, the number of ordinary differential equations (ODEs) in the model was first reduced from 12 to nine. Categorising the components in terms of their dynamic behaviour enabled us to further simplify the model down to five ODEs, and finally to a minimal model with two ODEs.

Like the original 12 ODE model, the 2 ODE model can generate delayed responses 
with different latencies. Thus, the basis of the time delay generation are specific interactions between the components, and intervening steps that contribute additive delays are not necessary. In the minimal model, the two ODEs represent the dynamics of active mGluRs and cytoplasmic $\mathrm{Ca}^{2+}$. The critical features of the model that underlie the delayed response are $\mathrm{Ca}^{2+}$ dependent negative feedback and autocatalysis.

The postulated requirement for autocatalysis and negative feedback can be tested experimentally. The interaction between $\mathrm{Ca}^{2+}$ and the receptors is mediated by PKC, and we predict that blocking PKC pharmacologically will prevent the generation of a delayed response. The dependence on autocatalysis is more difficult to study, given that the $\mathrm{Ca}^{2+}$ activation of $\mathrm{IP}_{3}$ receptors cannot currently be blocked without blocking $\mathrm{IP}_{3}$ dependent $\mathrm{Ca}^{2+}$ release completely. However, it should also be possible to measure the shape of the $\mathrm{Ca}^{2+}$ dependencies in experiments. In numerical simulations of the 2 ODE model, we did not manage to generate delayed responses unless the $\mathrm{Ca}^{2+}$ dependencies were described by sigmoidal Hill functions with cooperativities of at least 2. Given that there is no theoretical reason for this observation, the empirical simulation result should be compared with experimental data. It would be interesting, albeit technically challenging, to investigate systematically in experiments how the delayed $C a^{2+}$ response is influenced by interfering with the exact shape of the different $\mathrm{Ca}^{2+}$ dependencies.

One of the advantages of the minimal model is that it can be analysed mathematically, and that the behaviour of the system can be understood by plotting its temporal evolution in the phase plane. It was found that the activation of receptors by glutamate leads only to a small shift of the stable fixed point, but results in a large excursion of the state vector through the phase plane. Thus, by simplifying the complex Fiala model of mGluR dependent intracellular signalling in cerebellar Purkinje cells, we were able to show that the signalling network has the characteristic features of an excitable system (FitzHugh, 1960; Edelstein-Keshet, 1987).

In the temporal domain, the large excursion in the $\mathrm{Ca}^{2+}$ - receptor phase plane corresponds to the firing of a $\mathrm{Ca}^{2+}$ spike. The ability of the system to generate $\mathrm{Ca}^{2+}$ spikes could have several computational advantages. Unless the system is close to threshold, small perturbations in the concentrations of its components do not affect the decision whether or not a spike is generated. Fluctuations of the components have to cross thresh- 
old in order to have an effect on spiking; noise that stays below threshold does not influence the spike output and is ignored. Thus, excitability produces resistance to subthreshold noise. Furthermore, a spike based system provides a wide range of possible coding schemes that can be used, including simple rate codes where the information is represented by the number of spikes in a sampling interval, or temporal codes where the input is encoded by the timing of the spikes. Interestingly, it has been suggested previously that rate coded $\mathrm{Ca}^{2+}$ signals can be decoded by calmodulin (CaM) dependent kinase II (Putney, 1998; De Koninck \& Schulman, 1998).

Negative feedback and direct or indirect autocatalysis can be found in many intracellular pathways, and sigmoidal activation functions are ubiquitous in biochemical networks (Bray, 1995). Thus, the postulated delay generation mechanism might be a common feature of intracellular signalling systems. The generation of time delays plays an important part in many different processes such as rhythm generation (Goldbeter, 1995), auditory processing (Gerstner et al., 1996), classical conditioning (Fiala et al., 1996) and temporal pattern recognition (Hopfield, 1995).

Apart from generating time delays, intracellular signalling networks can have various other computational functions. It has been suggested that networks of proteins can perform logical operations (Bray, 1995), and that bistability in biochemical networks can be used to store information (Bhalla \& Iyengar, 1999). To fully understand neuronal information processing, it will therefore be necessary to understand the computations performed by the network of intracellular signalling components, and how this network interacts with the ion channels in the neuronal membrane. Given the complexity of intracellular networks, a multi-level modelling approach, where complex models are studied in parallel with simplified ones, seems to be the best strategy to pursue this goal.

\section{Acknowledgements}

V.S. is grateful for a University of Edinburgh Premier Scholarship and a Human Frontier

Science Program Organisation Long-Term Fellowship that provided his financial support. 


\section{Appendix: Mathematical Description of the Fiala Model}

In the Fiala model, PF input to the Purkinje cell results in activation of mGluRs by glutamate $([G l u])$. Activated receptors $B$ can be inactivated by dissociation of glutamate or by phosphorylation by PKC $(P)$, which gives rise to phosphorylated receptors $A$. Thus, the change of the concentrations of activated $(B)$ and phosphorylated $(A)$ mGluRs is given by

$$
\begin{gathered}
\frac{d B}{d t}=k_{1}\left(B_{\max }-B-A\right)[G l u]-k_{-1} B-k_{2} B P \\
\frac{d A}{d t}=k_{2} B P-k_{3} A
\end{gathered}
$$

where $B_{\max }$ is the total concentration of available mGluRs. A larger $B_{\max }$ results in faster production of activated receptors $B$, which speeds up the whole signalling cascade and shortens the time delay between mGluR activation and calcium release.

The activated mGluRs trigger the dissociation of a phospholipase C (PLC) specific Gprotein into subunits $\mathrm{G}_{\beta \gamma}$ and $\mathrm{G}_{P L C \alpha}$. Fiala et al. (1996) assume that the active subunit $\mathrm{G}_{P L C \alpha}$ can be inactivated by PKC phosphorylation and they represent the change of its concentration $G$ by:

$$
\frac{d G}{d t}=k_{4}\left(G_{\max }-G\right) B-k_{5} G-k_{6} G P
$$

The next step in the intracellular signalling cascade is the activation of PLC by $\mathrm{G}_{P L C \alpha}$ and the formation of inositol (1,4,5)-trisphosphate $\left(\mathrm{IP}_{3}\right)$ and diacylglycerol (DAG) from phosphatidylinositol (4,5)-bisphosphate $\left(\mathrm{PIP}_{2}\right)$. As there is also a $\mathrm{Ca}^{2+}$ dependent form of PLC (Mignery et al., 1992), the production of $\operatorname{IP}_{3}(I)$ and DAG $(D)$ is modelled as:

$$
\begin{gathered}
\frac{d I}{d t}=\left(I_{\max }-I\right)\left(k_{7} G+k_{8} P L C(C)\right)-k_{9} I \\
\frac{d D}{d t}=\left(D_{\max }-D\right)\left(k_{7} G+k_{8} P L C(C)\right)-k_{9} D
\end{gathered}
$$

with a $C a^{2+}(C)$ dependent PLC activity:

$$
P L C(C)=\frac{C^{2}}{C^{2}+K_{P L C}}
$$

$\mathrm{IP}_{3}$ activates $\mathrm{IP}_{3}$ receptor $\mathrm{Ca}^{2+}$ channels on the membrane of the endoplasmic reticulum (ER) leading to release of $\mathrm{Ca}^{2+}$ into the cytoplasm. In addition to the dependence on 
$\mathrm{IP}_{3}, \mathrm{Ca}^{2+}$ release through the $\mathrm{IP}_{3}$ receptors $\left(\mathrm{IP}_{3} \mathrm{Rs}\right)$ also shows a bell-shaped dependence on cytoplasmic $\mathrm{Ca}^{2+}$ (Bezprozvanny et al., 1991). In the model, the bell shaped $\mathrm{Ca}^{2+}$ dependence is based on the interaction of $\mathrm{Ca}^{2+}$ with two antagonistic binding sites, one with a stimulating effect on $\mathrm{IP}_{3} \mathrm{R}$ opening and one that inhibits it. The inhibitory site binds $\mathrm{Ca}^{2+}$ with a cooperativity of $n=1.65$ (Meissner et al., 1986). The binding and dissociation of $\mathrm{IP}_{3}$ is assumed to be much faster than all other reactions in the model. Thus, the $\mathrm{IP}_{3}$ binding is in equilibrium with respect to the $\mathrm{Ca}^{2+}$ binding and the concentration of open $\mathrm{IP}_{3} \mathrm{R}$ channels $R_{o}$ is given by

$$
R_{o}=\frac{I}{I+K_{I}} R_{a}
$$

where $R_{a}$ is the concentration of $\mathrm{IP}_{3} \mathrm{R}$ channels with $C a^{2+}$ bound to the stimulating, but not to the inhibitory site, and $\frac{I}{I+K_{I}}$ is the fraction of channels with bound $\mathrm{IP}_{3}$. Because of the fast $\mathrm{IP}_{3}$ binding, the concentrations of $\mathrm{Ca}^{2+}$ activated receptors $R_{a}$ and inhibited receptors $R_{i}$ can be modelled with two differential equations:

$$
\begin{gathered}
\frac{d R_{a}}{d t}=k_{12}\left(R_{\text {max }}-R_{a}-R_{i}\right) C-k_{13} R_{a}-k_{14} R_{a} C^{n}+k_{15} R_{i} \\
\frac{d R_{i}}{d t}=k_{14} R_{a} C^{n}-k_{15} R_{i}
\end{gathered}
$$

The $\mathrm{Ca}^{2+}$ which enters the cytoplasm through the $\mathrm{IP}_{3} \mathrm{Rs}$ is removed by the $\mathrm{Na}^{+} / \mathrm{Ca}^{2+}$ exchanger in the plasma membrane and the $\mathrm{Ca}^{2+}$ ATPase in the ER membrane. The change of the cytoplasmic $C a^{2+}$ concentration $C$ is given by the sum of three terms:

$$
\frac{d C}{d t}=\frac{d C}{d t}\left(I P_{3}\right)-\frac{d C}{d t}(\text { ATPase })-\frac{d C}{d t}(N a C a)
$$

The $\mathrm{IP}_{3}$ induced $\mathrm{Ca}^{2+}$ release increases linearly with the concentration of open $\mathrm{IP}_{3} \mathrm{Rs}$ $R_{o}$ and the $\mathrm{Ca}^{2+}$ concentration difference between ER and cytoplasm:

$$
\frac{d C}{d t}\left(I P_{3}\right)=k_{16} R_{o}\left(C_{E R}-C\right)
$$

The activity of the ER ATPase is modelled by a Hill function with a coefficient of 2 (Lytton et al., 1992):

$$
\frac{d C}{d t}(\text { ATPase })=k_{17} \frac{C^{2}}{C^{2}+K_{\text {ATPase }}}
$$


The $\mathrm{Na}^{+} / \mathrm{Ca}^{2+}$ exchanger carries $\mathrm{Na}^{+}$and $\mathrm{Ca}^{2+}$ ions with a stoichometry of 3:1 in opposite directions across the plasma membrane. The $\mathrm{Ca}^{2+}$ flux is outward if the cytoplasmic concentration is larger than the equilibrium concentration $C_{e q}$ (Carafoli, 1987):

$$
C_{e q}=C_{e x t} \frac{\left[N a^{+}\right]_{c y t}^{3}}{\left[N a^{+}\right]_{e x t}^{3}} \exp \left(\frac{V F}{R T}\right)
$$

where $C_{\text {ext }}$ and $\left[\mathrm{Na}^{+}\right]_{\text {ext }}$ are extracellular calcium and sodium concentrations, $\left[\mathrm{Na}^{+}\right]_{c y t}$ is the sodium concentration in the cytoplasm, $V$ represents the membrane voltage, $\mathrm{T}$ the thermodynamic temperature, $\mathrm{R}$ the gas constant and $\mathrm{F}$ the Faraday constant. The $\mathrm{Ca}^{2+}$ flux through the exchanger is modelled by (Hodgkin \& Nunn, 1987):

$$
\frac{d C}{d t}(N a C a)=k_{18} \frac{C-C_{e q}}{C-C_{e q}+K_{N a C a}}
$$

The rise in the cytoplasmic $\mathrm{Ca}^{2+}$ concentration triggers the activation of protein kinase $\mathrm{C}(\mathrm{PKC})$. PKC is transformed into its active form $(P)$ by binding one $\mathrm{Ca}^{2+}$ ion and one molecule of DAG $(D)$ :

$$
\frac{d P}{d t}=k_{10}\left(P_{\max }-P\right) D C-k_{11} P
$$

Two currents mediate the effect of $\mathrm{Ca}^{2+}$ on the membrane potential: an outward current through the $\mathrm{K}_{C a}$ channels and, for sufficiently elevated $\mathrm{Ca}^{2+}$ concentrations, an inward current through the $\mathrm{Na}^{+} / \mathrm{Ca}^{2+}$ exchanger. Thus, the change of membrane potential is described by

$$
\frac{d V}{d t}=k_{19} \frac{d C}{d t}(N a C a)-\bar{g}_{K C a} m_{K C a}(C, V)\left(V-V_{K}\right)-k_{20}\left(V-V_{b a s e}\right)
$$

where $V_{\text {base }}=-50 \mathrm{mV}$ is the elevated in vivo baseline potential and $k_{20}$ is given by the ratio of leakage conductance and membrane capacitance.

The outward current through the $K_{C a}$ channels is modelled as the product of three terms: the driving force given by the difference between membrane potential $V$ and potassium equilibrium potential $V_{K}=-85 \mathrm{mV}$, the maximum conductance $\bar{g}_{K C a}$, and the $\mathrm{Ca}^{2+}$ and voltage dependent activation variable $m_{K C a}(C, V)$. The activation of the channels by $\mathrm{Ca}^{2+}$ rise and membrane depolarisation is assumed to be instantaneous: 


$$
m_{K C a}(C, V)=m_{K C a, \infty}(C, V)=\frac{C^{2.6} s^{-1} S^{-1}}{C^{2.6}+\exp \left(\frac{11-V / m V}{22.5}\right) \mu M^{2.6}}
$$

The maximum conductance of the $\mathrm{K}_{C a}$ channels $\bar{g}_{K C a}$ is increased by PKC phosphorylation. Persistent phosphorylation of the $\mathrm{K}_{C a}$ channels by PKC requires simultaneous inhibition of PP-1. In analogy to one of the hypothesised mechanisms for AMPA receptor LTD (Ito \& Karachot, 1992; Linden \& Connor, 1993; Daniel et al., 1998; Schweighofer \& Ferriol, 2000), Fiala and collaborators have suggested that the inhibition of PP-1 by CF input is mediated by a signalling cascade through nitric oxide (NO) and cyclic guanosine monophosphate (cGMP). In their model, the CF evoked rise of cGMP in the Purkinje cell is described explicitly by:

$$
[c G M P]= \begin{cases}{[c G M P]_{\max }\left(\exp \left(\frac{t_{C F}-t}{\tau_{1}}\right)-\exp \left(\frac{t_{C F}-t}{\tau_{2}}\right)\right)} & \left(t>t_{C F}\right) \\ 0 & \left(t \leq t_{C F}\right)\end{cases}
$$

where $[c G M P]_{\max }$ is the maximum concentration of cGMP, $\tau_{1}$ and $\tau_{2}$ are the decay and rise time constants and $t_{C F}$ is the onset of the cGMP rise. The cGMP rise in the Purkinje cell cytoplasm activates protein kinase $\mathrm{G}$ (PKG) which phosphorylates and activates the PP-1 inhibitor G-substrate. The resulting change of the $\mathrm{K}_{C a}$ channel conductance $\bar{g}_{K C a}$ is given by:

$$
\frac{d \bar{g}_{K C a}}{d t}=k_{23}\left(\bar{g}_{K C a, \max }-\bar{g}_{K C a}\right) P[c G M P]-k_{24} \bar{g}_{K C a} N
$$

The second term in Eq. 36 describes the $\mathrm{K}_{C a}$ channel dephosphorylation caused indirectly via PP-1 activation and G-substrate inhibition by the protein phosphatase calcineurin $(N)$. Calcineurin is activated by $\mathrm{Ca}^{2+} /$ calmodulin with a cooperativity of approximately 3 (Stemmer \& Klee, 1994). Thus, the concentration change of active calcineurin $N$ is modelled by:

$$
\frac{d N}{d t}=k_{21}\left(N_{\max }-N\right) C^{3}-k_{22} N
$$

The network of interactions between the components of the Fiala model is summarised in figure 1. 


\section{References}

Ahissar, E., \& Arieli, A. (2001). Figuring space by time. Neuron, 32, 185-201.

Batchelor, A. M., \& Garthwaite, J. (1993). Novel synaptic potentials in cerebellar Purkinje cells: probable mediation by metabotropic glutamate receptors. Neuropharmacology, 32, 11-20.

Batchelor, A. M., \& Garthwaite, J. (1997). Frequency detection and temporally dispersed synaptic signal association through a metabotropic glutamate receptor pathway. Nature, 385, 74-77.

Batchelor, A. M., Madge, D. J., \& Garthwaite, J. (1994). Synaptic activation of metabotropic glutamate receptors in the parallel fibre-Purkinje cell pathway in rat cerebellar slices. Neuroscience, 63, 911-915.

Bezprozvanny, I., Watras, J., \& Ehrlich, B. E. (1991). Bell-shaped calcium-dependent curves of Ins(1,4,5)-P3 gated and calcium gated channels from endoplasmic reticulum of cerebellum. Nature, 351, 751-754.

Bhalla, U. S., \& Iyengar, R. (1999). Emergent properties of networks of biological signaling pathways. Science, 283, 381-387.

Bialek, W., Rieke, F., de Ruyter van Steveninck, R., \& Warland, D. (1991). Reading a neural code. Science, 252, 1854-1857.

Bray, D. (1995). Protein molecules as computational elements in living cells. Nature, 376, $307-312$.

Carafoli, E. (1987). Intracellular calcium homeostasis. Ann. Rev. Biochem., 56, 395-433.

Daniel, H., Levenes, C., \& Crépel, F. (1998). Cellular mechanisms of cerebellar LTD. Trends Neurosci., 21, 401-407.

De Boer, R. J. (1983). GRIND. GReat INtegrator Differential equations User Manual. Theoretical Biology, Utrecht University, The Netherlands. 
De Koninck, P., \& Schulman, H. (1998). Sensitivity of CaM kinase II to the frequency of Ca2+ oscillations. Science, 279, 227-230.

deCharms, R. C., \& Zador, A. (2000). Neural representation and the cortical code. Annu. Rev. Neurosci., 23, 613-647.

Edelstein-Keshet, L. (1987). Mathematical Models in Biology. McGraw-Hill Inc., New York.

Eurich, C. W., Cowan, J. D., \& Milton, J. G. (1997). Hebbian delay adaptation in a network of integrate-and-fire neurons. In Gerstner, W., Germond, A., Hasler, M., \& Nicoud, J. D. (Eds.), Proceedings of the 7th International Conference on Artifical Neural Networks ICANN 9\%, pp. 157-162. Springer Verlag, Berlin.

Eurich, C. W., Ernst, U., \& Pawelzik, K. (1998). Continous dynamics of neuronal delay adaptation. In Niklasson, L., Bodén, M., \& Ziemke, T. (Eds.), Proceedings of the 8th International Conference on Artifical Neural Networks ICANN 98, pp. 355-360. Springer Verlag, Berlin.

Fiala, J. C., Grossberg, S., \& Bullock, D. (1996). Metabotropic glutamate receptor activation in cerebellar Purkinje cells as substrate for adaptive timing of the classically conditioned eye-blink response. J. Neurosci., 16, 3760-3774.

Finch, E. A., \& Augustine, G. J. (1998). Local calcium signalling by inositol-1,4,5trisphosphate in Purkinje cell dendrites. Nature, 396, 753-756.

FitzHugh, R. (1960). Thresholds and plateaus in the Hodgkin-Huxley nerve equations. J. Gen. Physiol., 43, 867-896.

Gerstner, W., Kempter, R., van Hemmen, J., \& Wagner, H. (1996). A neuronal learning rule for sub-millisecond temporal coding. Nature, 383, 76-78.

Goldbeter, A. (1995). A model for circadian oscillations in the Drosophila period protein (PER). Proc. R. Soc. Lond. B, 261, 319-324.

Hodgkin, A. L., \& Nunn, B. J. (1987). The effect of ions on sodium-calcium exchange in salamander rods. J. Physiol., 391, 371-398. 
Hopfield, J. J. (1995). Pattern recognition computation using action potential timing for stimulus representation. Nature, 376, 33-36.

Hüning, H., Glünder, H., \& Palm, G. (1998). Synaptic delay learning in pulse-coupled neurons. Neural Comp., 10, 555-565.

Huxter, J., Burgess, N., \& O'Keefe, J. (2003). Independent rate and temporal coding in hippocampal pyramidal cells. Nature, 425, 828-832.

Ito, M., \& Karachot, L. (1992). Protein kinases and phosphatase inhibitors mediating long-term desensitization of glutamate receptors in cerebellar Purkinje cells. Neuroscience Res., 14, 27-38.

Kawabata, S., Tsutsumi, R., Kohara, A., Yamaguchi, T., Nakanishi, S., \& Okada, M. (1996). Control of calcium oscillations by phosphorylation of metabotropic glutamate receptors. Nature, 383, 89-92.

Laurent, G. (1996). Dynamical representation of odors by oscillating and evolving neural assemblies. Trends Neurosci., 19, 489-496.

Linden, D. J., \& Connor, J. A. (1993). Cellular mechanisms of long-term depression in the cerebellum. Curr. Opin. Neurobiol., 3, 401-406.

Lytton, J., Westlin, M., Burk, S. E., Shull, G. E., \& MacLennan, D. H. (1992). Functional comparisons between isoforms of the sarcoplasmic or endoplasmic reticulum family of calcium pumps. J. Biol. Chem., 267, 14483-14489.

Meissner, G., Darling, E., \& Eveleth, J. (1986). Kinetics of rapid $C a^{2+}$ release by sarcoplasmic reticulum. Effects of $\mathrm{Ca}^{2+}, \mathrm{Mg}^{2+}$ and adenine nucleotides. Biochemistry, 25, 236-244.

Mignery, G. A., Johnston, P. A., \& Südhof, T. C. (1992). Mechanism of $C a^{2+}$ inhibition of inositol 1,4,5-trisphosphate $\left(\operatorname{Ins}_{3}\right)$ binding to the cerebellar $\operatorname{Ins} P_{3}$ receptor. $J$. Biol. Chem., 267, 7450-7455.

Napp-Zinn, H., Jansen, M., \& Eckmiller, R. (1996). Recognition and tracking of impulse patterns with delay adaptation in biology-inspired pulse processing neural net (BPN) hardware. Biol. Cybernetics, 74, 449-453. 
Natschläger, T., \& Ruf, B. (1998). Spatial and temporal pattern analysis via spiking neurons. Network, 9, 319-332.

Nishizuka, Y. (1988). The molecular heterogeneity of protein kinase C and its implications for cellular regulation. Nature, 334, 661-665.

Putney, J. W. (1998). Calcium signaling: Up, down, up, down... What's the point?. Science, 279, 191-192.

Rieke, F., Warland, D., de Ruyter van Steveninck, R., \& Bialek, W. (1997). Spikes: Exploring the neural code. MIT Press, Cambridge, Massachusetts.

Schweighofer, N., \& Ferriol, G. (2000). Diffusion of nitric oxide can facilitate cerebellar learning: A simulation study. Proc. Natl. Acad. Sci. USA, 97, 10661-10665.

Stemmer, P. M., \& Klee, C. B. (1994). Dual calcium ion regulation of calcineurin by calmodulin and calcineurin B. Biochemistry, 33, 6859-6866.

Steuber, V., \& Willshaw, D. J. (1997). How a single Purkinje cell could learn the adaptive timing of the classically conditioned eye-blink response. In Gerstner, W., Germond, A., Hasler, M., \& Nicoud, J. D. (Eds.), Proceedings of the 7th International Conference on Artifical Neural Networks ICANN 97, pp. 115-120. Springer Verlag, Berlin.

Steuber, V., \& Willshaw, D. J. (1999). Adaptive leaky integrator models of cerebellar Purkinje cells can learn the clustering of temporal patterns. Neurocomputing, 26, $271-276$.

Steuber, V., \& Willshaw, D. J. (2004). A biophysical model of synaptic delay learning and temporal pattern recognition in a cerebellar Purkinje cell. J. Comp. Neurosci., $17,149-164$.

Tempia, F., Miniaci, M. C., Anchisi, D., \& Strata, P. (1998). Postsynaptic currents mediated by metabotropic glutamate receptors in cerebellar Purkinje cells. J. Neurophysiol., 80, 520-528.

Thompson, R. F., \& Krupa, D. J. (1994). Organization of memory traces in the mammalian brain. Ann. Rev. Neurosci., 17, 519-549. 
Thorpe, S., Fize, F., \& Marlot, C. (1996). Speed of processing in the human visual system. Nature, 381, 520-522.

Yarfitz, S., \& Hurley, J. B. (1994). Transduction mechanisms of vertebrate and invertebrate photoreceptors. J. Biol. Chem., 269, 14329-14332.

Yeo, C. H., \& Hesslow, G. (1998). Cerebellum and conditioned reflexes. Trends Cognitive Sci., 2, 322-330. 


\section{Tables}

\section{Table 1: Parameters of the 5 ODE model}

\begin{tabular}{|c|c|c|}
\hline Parameter & Description & Value \\
\hline$k_{1}$ & rate constant for glutamate binding to mGluRs & $0.1 \mu M^{-1} s^{-1}$ \\
\hline$k_{-1}$ & rate constant for glutamate dissociation from mGluRs & $0.01 s^{-1}$ \\
\hline$k_{2}$ & rate constant for PKC phosphorylation of mGluRs & $4.0 \mu M^{-1} s^{-1}$ \\
\hline$k_{7}$ & rate constant for $\mathrm{G}$-protein activated formation of $\mathrm{IP}_{3} / \mathrm{DAG}$ & $0.2 \mu M^{-1} s^{-1}$ \\
\hline$k_{8}$ & rate constant for $\mathrm{Ca}^{2+}$ activated formation of $\mathrm{IP}_{3} / \mathrm{DAG}$ & $40.0 s^{-1}$ \\
\hline$k_{9}$ & rate constant for $\mathrm{IP}_{3} / \mathrm{DAG}$ degradation & $80.0 s^{-1}$ \\
\hline$k_{12}$ & rate constant for $\mathrm{Ca}^{2+}$ activation of $\mathrm{IP}_{3} \mathrm{Rs}$ & $60.0 \mu M^{-1} s^{-1}$ \\
\hline$k_{13}$ & rate constant for dissociation of $\mathrm{Ca}^{2+}$ from activated $\mathrm{IP}_{3} \mathrm{Rs}$ & $48.6 s^{-1}$ \\
\hline$k_{14}$ & rate constant for $\mathrm{Ca}^{2+}$ inactivation of $\mathrm{IP}_{3} \mathrm{Rs}$ & $7.55 \mu M^{-1.65} s^{-1}$ \\
\hline$k_{15}$ & rate constant for dissociation of $\mathrm{Ca}^{2+}$ from inactivated $\mathrm{IP}_{3} \mathrm{Rs}$ & 0.0 \\
\hline$k_{16}$ & rate constant for release of $\mathrm{Ca}^{2+}$ from $\mathrm{ER}$ & $2.0 \mu M^{-1} s^{-1}$ \\
\hline$k_{17}$ & rate constant for removal of $\mathrm{Ca}^{2+}$ by the ATPase & $50.0 \mu M s^{-1}$ \\
\hline$K_{C}$ & activation constant for $\mathrm{Ca}^{2+}$ activation of PLC & $20.0 \mu M^{2}$ \\
\hline$K_{I}$ & activation constant for $\mathrm{IP}_{3}$ activation of $\mathrm{IP}_{3} \mathrm{Rs}$ & $0.2 \mu M$ \\
\hline$K_{\text {ATPase }}$ & binding constant for $\mathrm{Ca}^{2+}$ binding to the ATPase & $0.2 \mu M^{2}$ \\
\hline$B_{\max }$ & total mGluR concentration & $20.0 \mu M$ \\
\hline$I_{\max }$ & total (bound and unbound) $\mathrm{IP}_{3}$ concentration & $1.0 \mu M$ \\
\hline$R_{\max }$ & total concentration of available $\mathrm{IP}_{3} \mathrm{Rs}$ & $1.0 \mu M$ \\
\hline$C_{E R}$ & reticular $C a^{2+}$ concentration & $1 m M$ \\
\hline
\end{tabular}




\section{Table 2: Parameters of the 2 ODE model}

\begin{tabular}{|l|l|l|}
\hline Parameter & Description & Value \\
\hline \hline$k_{a}$ & rate constant for glutamate activation of mGluRs & $0.00125 \mu M^{-1} s^{-1}$ \\
\hline$k_{b}$ & rate constant for glutamate dissociation from mGluRs & $0.0025 s^{-1}$ \\
\hline$k_{c}$ & rate constant for $C a^{2+}$ dependent inactivation of mGluRs & $0.25 s^{-1}$ \\
\hline$k_{d}$ & rate constant for $C a^{2+}$ release from intracellular stores & $0.25 s^{-1}$ \\
\hline$k_{e}$ & rate constant for $C a^{2+}$ uptake into intracellular stores & $2.5 \mu M s^{-1}$ \\
\hline$K_{a}$ & Hill constant for $C a^{2+}$ dependent inactivation of mGluRs & $1.2 \mu M$ \\
\hline$K_{b}$ & Hill constant for $C a^{2+}$ release from intracellular stores & $1.2 \mu M$ \\
\hline$K_{c}$ & Hill constant for $C a^{2+}$ uptake into intracellular stores & $2.0 \mu M$ \\
\hline$B_{\max }$ & total mGluR concentration & $30-180 \mu M$ \\
\hline$n$ & Hill coefficient for $C a^{2+}$ dependent processes & 4.0 \\
\hline
\end{tabular}




\section{Figures}

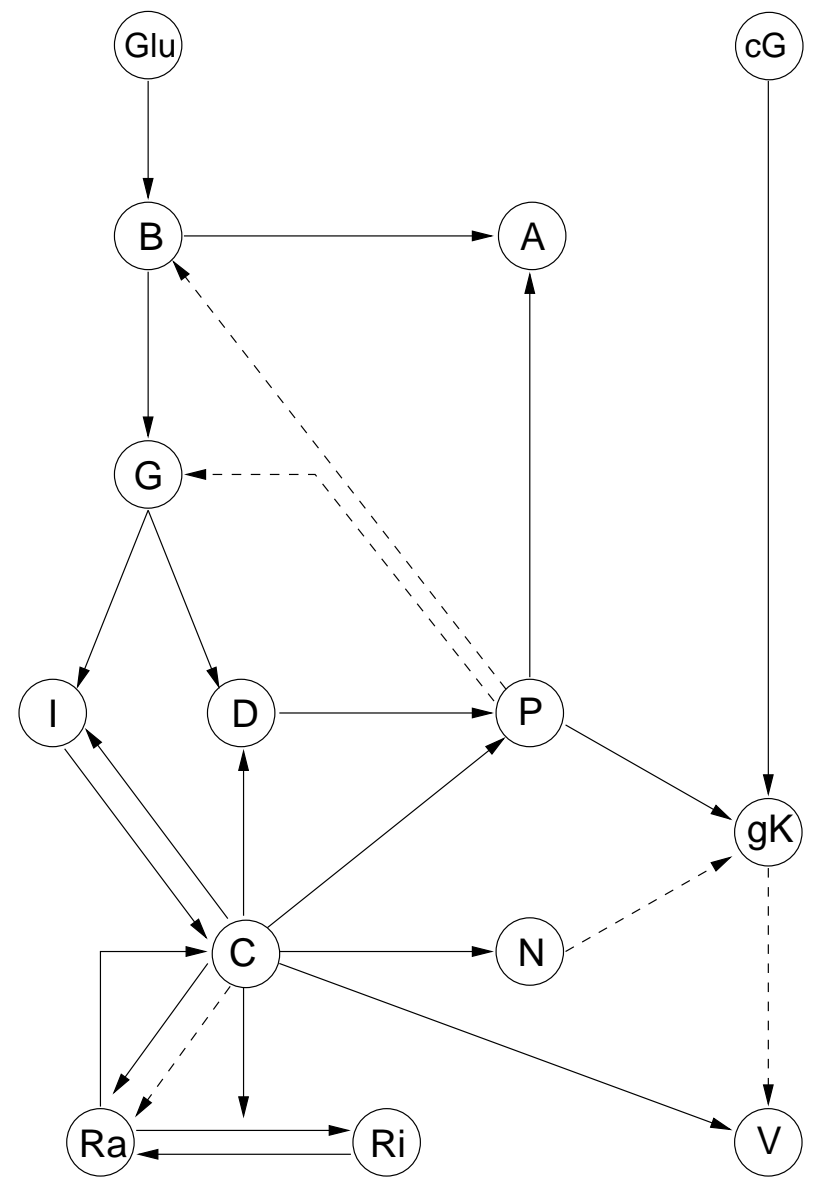

Figure 1: Diagram summarising the interactions between the intracellular signalling components in the Fiala model (Fiala et al., 1996). Solid arrows between two components indicate that an increase in the first component results in an increase in the second component. Broken arrows indicate interactions where an increase in the first component triggers a decrease in the second one. The individual components are: Glu glutamate, cG cGMP, B activated mGluRs, A inactivated mGluRs, G activated G-protein $\mathrm{G}_{P L C \alpha}$, I $\mathrm{IP}_{3}, \mathrm{D}$ DAG, $\mathrm{P}$ protein kinase $\mathrm{C}, \mathrm{C}$ calcium, $\mathrm{R}_{a}$ activated $\mathrm{IP}_{3}$ receptors, $\mathrm{R}_{i}$ inactivated $\mathrm{IP}_{3}$ receptors, $\mathrm{N}$ calcineurin, gK maximum KCa channel conductance and $\mathrm{V}$ membrane voltage. 
(a)

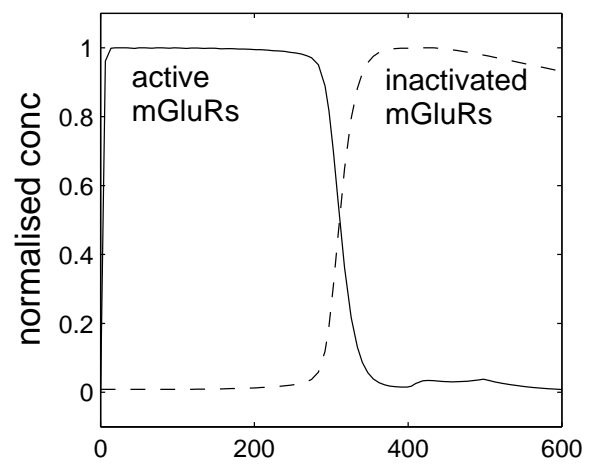

(c)

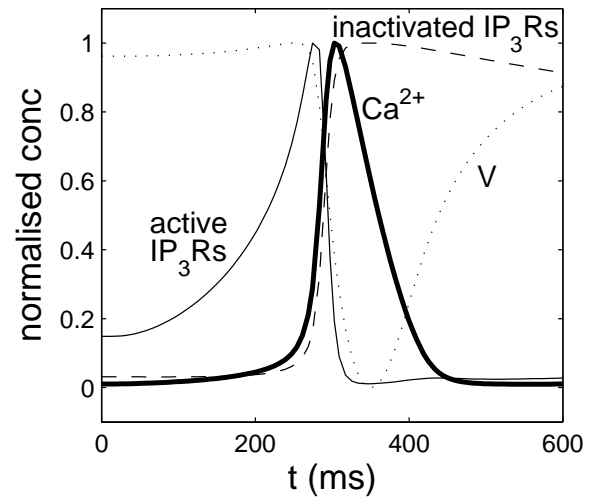

(b)

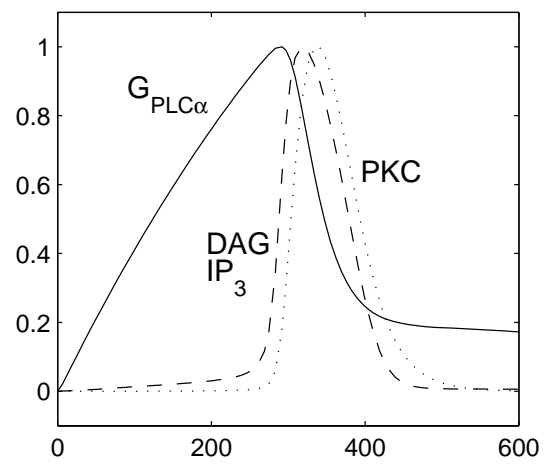

(d)

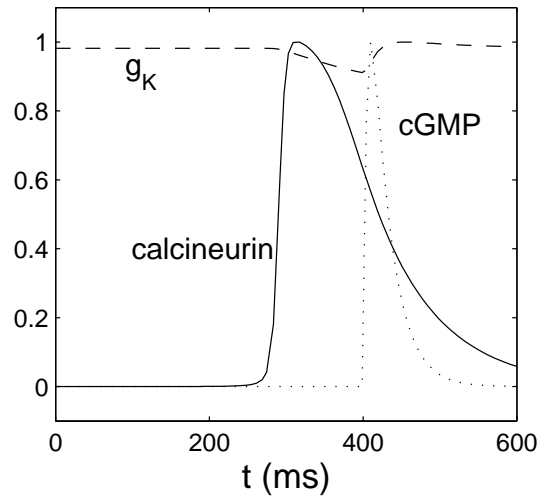

Figure 2: Behaviour of the Fiala model in response to a $10 \mu M$ glutamate pulse from $t=0$ to $t=500 \mathrm{~ms}$. For easier comparison, all concentrations $X$ and the voltage $V$ are normalised by plotting $X / \max (X)$ and $(V-\min (V)) /(\max (V)-\min (V))$, respectively. (a) activated mGluRs (solid) and inactivated mGluRs (dashed). (b) $\mathrm{G}_{P L C \alpha}$ (solid), $\mathrm{IP}_{3}$ (dashed) and PKC (dotted). (c) active $\mathrm{IP}_{3}$ receptors (thin solid line), inactive $\mathrm{IP}_{3}$ receptors (dashed), $\mathrm{Ca}^{2+}$ (thick solid line) and the membrane voltage $(V$, dotted). (d) calcineurin (solid), $\bar{g}_{K C a}$ (dashed), and cGMP (dotted). The extreme values of the different variables are: $\max (B)=64.044 \mu M, \max (A)=65.624 \mu M, \max (G)=0.478 \mu M$, $\max (I)=0.259 \mu M, \max (C)=0.972 \mu M, \max \left(R_{a}\right)=0.521 \mu M, \max \left(R_{i}\right)=0.995 \mu M$, $\max (C)=6.765 \mu M, \max (V)=-45.46 m V, \min (V)=-50.03 m V, \max (N)=$ $1.918 \mu M, \max \left(\bar{g}_{K C a}\right)=0.076 S, \max ([c G M P])=2.646 \mu M$. All parameters are as in Fiala et al. (1996), with the exception of the concentration of available mGluRs, which is set to $B_{\max }=66.5 \mu M$. 
(a)

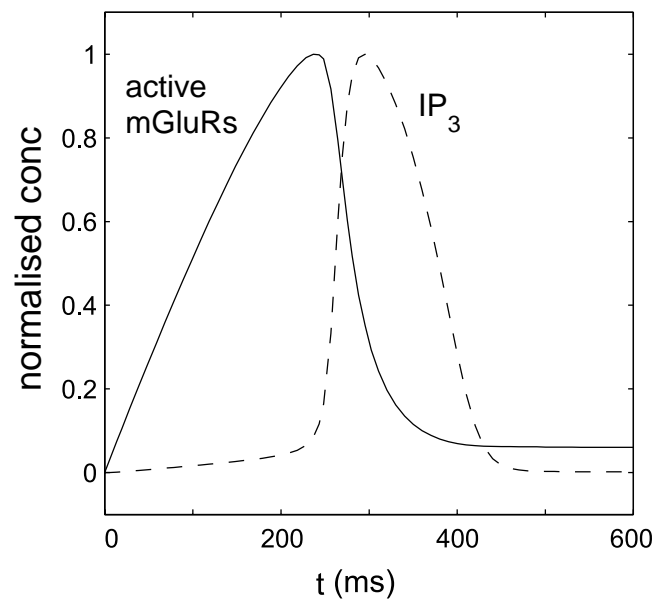

(b)

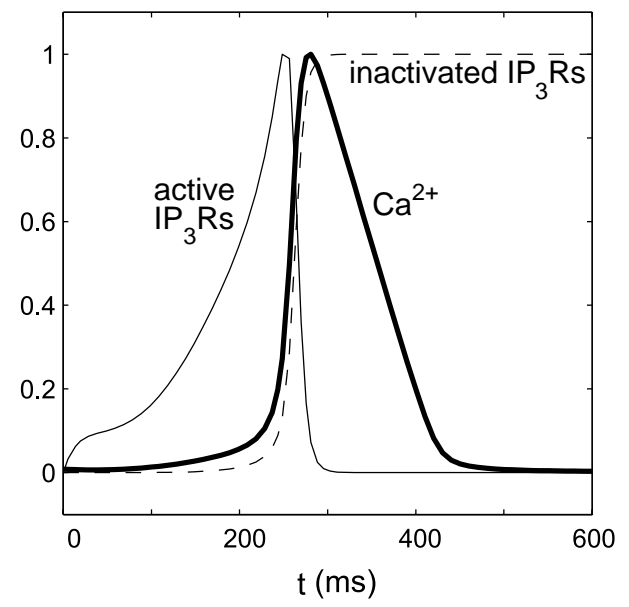

Figure 3: Behaviour of the 5 ODE model in response to a $10 \mu M$ glutamate pulse from $t=0$ to $t=500 \mathrm{~ms}$. A concentration of available mGluRs $B_{\max }=20 \mu \mathrm{M}$ implements a time delay of $\Delta t \approx 300 \mathrm{~ms}$. (a) active mGluRs (solid) and $\mathrm{IP}_{3}$ (dashed). (b) active $\mathrm{IP}_{3}$ receptors (thin solid line), inactive $\mathrm{IP}_{3}$ receptors (dashed) and $\mathrm{Ca}^{2+}$ (thick solid line). For easier comparison, all concentrations $X$ are normalised by plotting $X / \max (X)$. The maximum values of the variables are: $\max (B)=3.657 \mu M, \max (I)=0.255 \mu M$, $\max \left(R_{a}\right)=0.507 \mu M, \max \left(R_{i}\right)=1.00 \mu M, \max (C)=6.931 \mu M$. Simulation parameters are given in table 1.
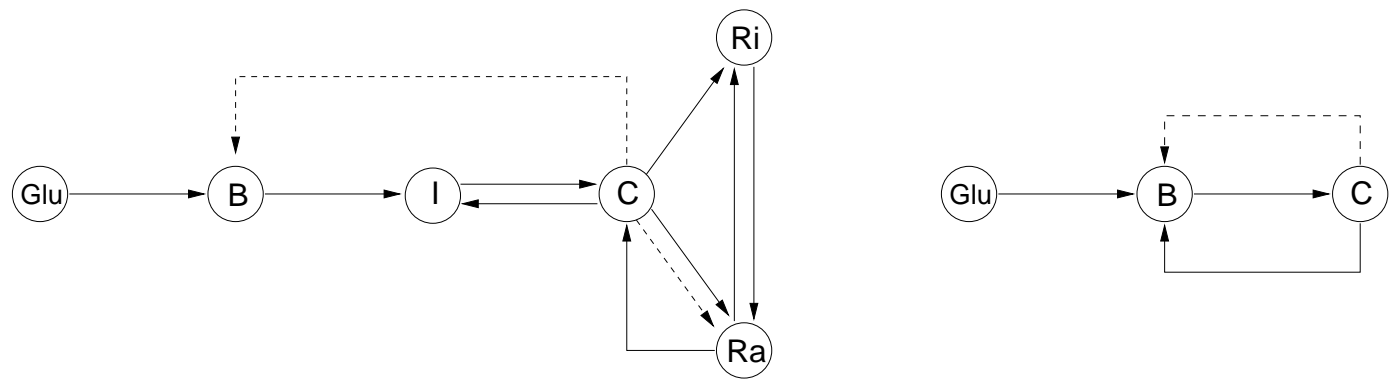

Figure 4: Diagram summarising the interactions between the intracellular signalling components in the 5 ODE model (left) and the minimal 2 ODE model (right). Solid and broken arrows represent positive and negative effects of a component on another one, respectively. The components are: Glu glutamate, $\mathrm{B}$ active mGluRs, $\mathrm{I} \mathrm{IP}_{3}, \mathrm{C}$ calcium, $\mathrm{R}_{a}$ calcium-activated $\mathrm{IP}_{3}$ receptors and $\mathrm{R}_{i}$ calcium-inactivated $\mathrm{IP}_{3}$ receptors. 
(a)

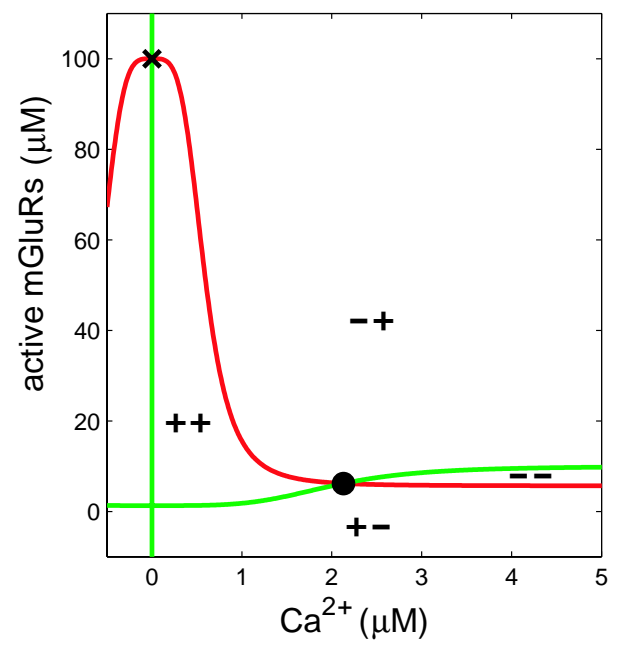

(b)

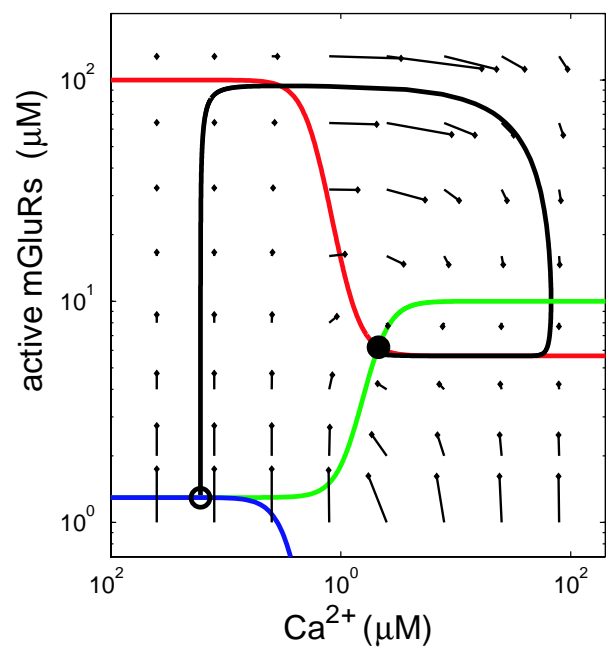

Figure 5: Phase plane diagrams for the minimal model with two ODEs representing the dynamics of active mGluRs $B$ and $C a^{2+} C$. (a) B-nullcline (red) and C-nullclines (green) of the model for a glutamate concentration $G_{2}=10.0 \mu M$ and the parameter values in table 2. The system has a saddle point at the intersection of the B-nullcline with the Baxis (represented by $\mathrm{x}$ ), and a stable fixed point at the intersection of the B-nullcline with the second C-nullcline (the filled circle in the figure). The nullclines divide the phase plane into four regions with different orientations of the direction vector $\{\dot{B}, \dot{C}\}:(++)$ indicates $\dot{B}>0$ and $\dot{C}>0,(-+) \dot{B}<0$ and $\dot{C}>0,(--) \dot{B}<0$ and $\dot{C}<0$ and $(+-) \dot{B}>0$ and $\dot{C}<0$. (b) Response of the model to a transient increase of the glutamate concentration [Glu] from $G_{1}=0.02185 \mu M$ to $G_{2}=10.0 \mu M$. The B-nullclines for $G_{1}=0.02185 \mu M$ and $G_{2}=10.0 \mu M$ are indicated in blue and red, repectively. The C-nullcline is shown in green. The empty and the filled circle mark the location of the stable fixed points $\mathbf{S}^{0}$ for $[G l u]=G_{1}$ and $\mathbf{S}^{*}$ for $[G l u]=G_{2}$. The arrows indicate the direction vectors $\{\dot{B}, \dot{C}\}$ at different values of $\{B, C\}$. For clarity, each direction vector has been scaled by $50 \%$ and points from $\{B, C\}$ to $\{B+\dot{B} / 2, C+\dot{C} / 2\}$ (note that the plot is double logarithmic). In response to the $G_{1} \rightarrow G_{2}$ step, the state vector $\mathbf{S}=\{B, C\}$ travels from $\mathbf{S}^{0}$ to $\mathbf{S}^{*}$ through the phase plane (thick solid line). 
(a)

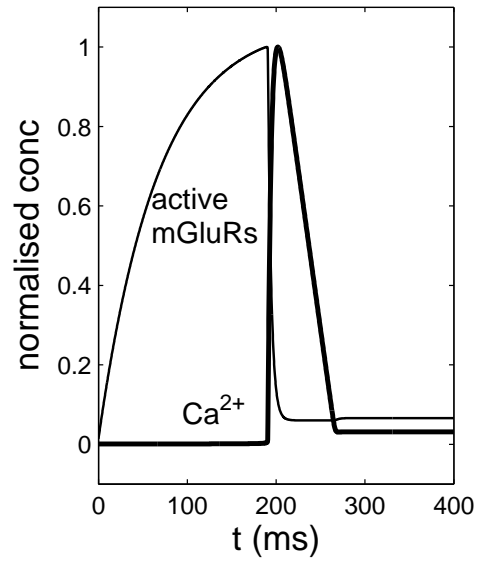

(b)

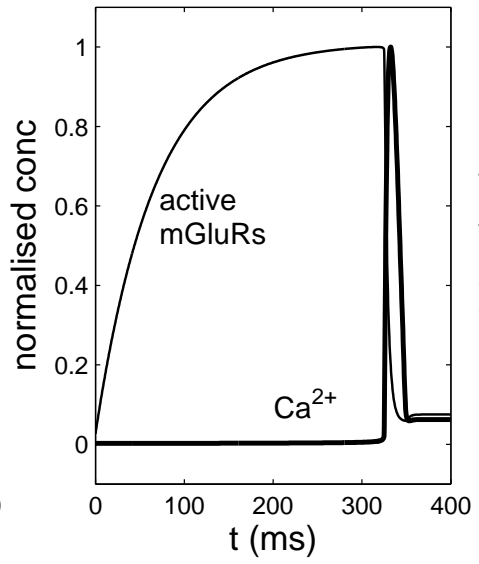

(c)

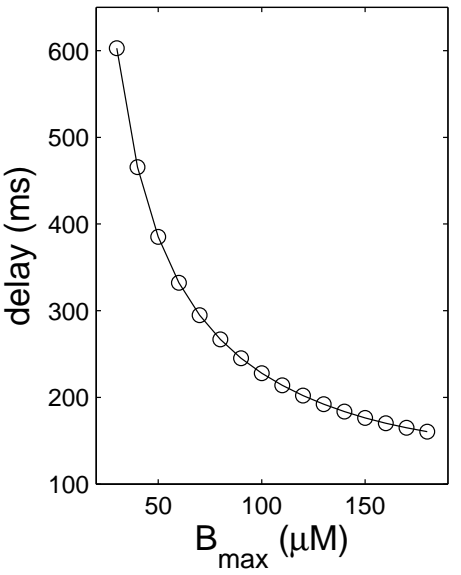

Figure 6: Dependence of the time delay between mGluR activation and $\mathrm{Ca}^{2+}$ response in the minimal model on the concentration of available receptors $B_{\max }$. (a), (b) Temporal evolution of the concentrations of active mGluRs (thin line) and $\mathrm{Ca}^{2+}$ (thick line) in response to a step increase in glutamate concentration from $G_{1}=0.02185 \mu M$ to $G_{2}=$ 10.0 $\mu M$ at $t=0$. The concentrations of available receptors $B_{\max }$ in (a) and (b) are $120 \mu M$ and $60 \mu M$, respectively (other parameter values as in figure 5). For easier comparison, the $\mathrm{Ca}^{2+}$ and receptor concentrations have been normalised to their maximum values. (c) Larger concentrations of available receptors $B_{\max }$ result in shorter time delays. 